\title{
ON A CONJECTURE OF WOOD
}

\author{
KAZUHIRO KAWAMURA \\ Institute of Mathematics, University of Tsukuba, Tsukuba, Ibaraki 305-8071, Japan \\ e-mail:kawamura@math.tsukuba.ac.jp
}

(Received 1 September, 2003; accepted 7 July, 2004)

\begin{abstract}
We show that there exists a locally compact separable metrizable space $L$ such that $C_{0}(L)$, the Banach space of all continuous complex-valued functions vanishing at infinity with the supremum norm, is almost transitive. Due to a result of Greim and Rajagopalan [3], this implies the existence of a locally compact Hausdorff space $\tilde{L}$ such that $C_{0}(\tilde{L})$ is transitive, disproving a conjecture of Wood [9]. We totally owe our construction to a topological characterization due to Sánches [8].
\end{abstract}

2000 Mathematics Subject Classification. 54C35, 46B04, 54G99.

1. Introduction, main theorem and preliminaries. For a locally compact Hausdorff space $L, C_{0}(L)$ denotes the Banach space of all continuous complex-valued functions on $L$ vanishing at infinity, equipped with the supremum norm. A Banach space $X$ is said to be transitive (resp. almost transitive) if the isometry group $G(X)$ acts transitively on the unit sphere $S(X)=\{x \in X \mid\|x\|=1\}$ (resp. the orbit $G(X) \cdot x$ is dense in $S(X)$ for each $x \in S(X)$ ). In [9], Wood conjectured that $C_{0}(L)$ is not transitive for any locally compact Hausdorff space $L$ unless $L$ is a singleton. In [3], the conjecture was verified for the Banach space $C_{0}(L: \mathbf{R})$ of all real-valued continuous functions on $L$ vanishing at infinity. Greim and Rajagopalan proved in [3] that the existence of a locally compact Hausdorff space $L$ with $C_{0}(L)$ being almost transitive implies the existence of a locally compact Hausdorff space $\tilde{L}$ such that $C_{0}(\tilde{L})$ is transitive. In [7], the verification of the conjecture was reduced to the case in which $L$ has the metrizable onepoint compactification $\alpha L$. Furthermore, Sánches in [8] gave an explicit topological characterization of the space $L$ with the almost transitive $C_{0}(L)$. Theorem 2 and 3 of [8] are restated below. Following [8], we say that a locally compact Hausdorff space $L$ is an Wood space (resp. an almost Wood space) if $C_{0}(L)$ is transitive (resp. almost transitive).

THEOREM 1.1 ([8, Theorem 2,3]). Let L be a locally compact metrizable space such that the one-point compactification $\alpha L=L \cup\{\infty\}$ is metrizable and $\operatorname{dim} \alpha L=1$. Then $L$ is an almost Wood space if and only if $L$ satisfies the following condition:

for each pair of sequences $\left\{E_{i} \mid i=0, \ldots, n\right\}$ and $\left\{F_{i} \mid i=0, \ldots, n\right\}$ of compact subsets of $\alpha L$ satisfying

(1) $\alpha L=\cup_{i=0}^{n} E_{i}=\cup_{i=0}^{n} F_{i}$,

(2) the point of infinity $\infty \in E_{0} \cap F_{0}$, and

(3) $E_{i} \cap E_{j}=F_{i} \cap F_{j}=\emptyset$ if $|i-j|>1$,

there exists a homeomorphism $\varphi: \alpha L \rightarrow \alpha L$ such that $\varphi(\infty)=\infty$ and $\varphi\left(E_{i}\right) \subset F_{i-1} \cup F_{i} \cup F_{i+1}$ for each $i=0, \ldots, n$, where $F_{-1}=F_{n+1}=\emptyset$. 
The clear characterization above led us to find out a non-trivial almost Wood space, and hence, by [3], a non-trivial Wood space.

The almost Wood space $L$ given in this note is (the pseudo-arc) $\backslash$ a singleton\}. The pseudo-arc $P$ is the topologically unique compact connected metric space satisfying the following two conditions (a) and (b). A chain of a metric space $X$ is an open cover $C=\{C(0), \ldots, C(n)\}$ of $X$ such that $C(i) \cap C(j) \neq \emptyset$ if and only if $|i-j| \leq 1$. Each member $C(i)$ of a chain $C=\{C(0), \ldots, C(n)\}$ is called a link. Also we fix a metric on $X$ and let $\operatorname{mesh} C=\max _{i=0, \ldots, n} \operatorname{diam} C(i)$, where $\operatorname{diam} C(i)$ is the diameter of $C(i)$ with respect to the metric.

(a) For each $\epsilon>0$, there exists a chain $C$ such that mesh $C<\epsilon$.

(b) For each pair of points $x, y \in P$, there exists a homeomorphism $\varphi: P \rightarrow P$ such that $\varphi(x)=y$.

The space was constructed by R. H. Bing [1] and E. E. Moise [6]. It has been playing an important role in continuum theory (in topology) and our result is another simple application of the topology of the pseudo-arc.

Main theorem. Let $P$ be the pseudo-arc, $p \in P$ and let $L=P \backslash\{p\}$. Then $C_{0}(L)$ is almost transitive.

In the rest of this section, we recall some properties of the pseudo-arc. [5] is an excellent survey article on the space, from which we quote all results below. First observe that $\operatorname{dim} P=1$ by the above condition (a) and the connectedness of $P$.

Definition 1.2. (1) A function $f:\{0,1, \ldots, m\} \rightarrow\{0, \ldots, n\}$ is called a pattern if $|f(i)-f(i+1)| \leq 1$ for each $i=0, \ldots, m-1$.

(2) Let $C=\{C(0), \ldots, C(n)\}$ and $D=\{D(0), \ldots, D(m)\}$ be chains of the pseudoarc. The chain $D$ is said to follow a pattern $f$ in the chain $C$ if $\operatorname{cl} D(i) \subset C(f(i))$ for each $i=0, \ldots, m$. Here $\operatorname{cl} D(i)$ denotes the closure of $D(i)$.

THEOREM 1.3 ([5, p. 108]). For each point $p \in P$ and for each $\epsilon>0$, there exists a chain $C=\{C(0), \ldots, C(n)\}$ such that mesh $C<\epsilon$ and $p \in C(0) \backslash \cup_{i=1}^{n} C(i)$.

The following is an immediate consequence of [5, p. 108, Theorem[60]], being applied to $j=i_{0}=0$, for which the hypothesis of the theorem is automatically satisfied.

THeOREM 1.4. Let $p$ be a point of the pseudo-arc $P$ and let $C=\{C(0), \ldots, C(n)\}$ be a chain of $P$ such that $p \in C(0)$. For each pattern $f:\{0, \ldots, m\} \rightarrow\{0, \ldots, n\}$ with $f(0)=0$, there exists a chain $D=\{D(0), \ldots, D(m)\}$ which follows $f$ and $p \in D(0)$.

The following theorem, here stated for the pseudo-arc only, provides us with the standard method to construct homeomorphisms of the pseudo-arc. The proof of main theorem given in the next section is a simple application of Theorem 1.5 below to a specific situation.

For a chain $C=\{C(0), \ldots C(n)\}$ of the pseudo-arc $P$ and for a point $p \in P, \operatorname{st}(p, C)$ denotes the collection of links containing $p$. Note that $\operatorname{st}(p, C)$ consists of at most two links.

THEOREM 1.5 ([5, p. 109]). Let $\left\{C_{i} \mid i=0,1, \ldots\right\}$ and $\left\{D_{i} \mid i=0,1, \ldots\right\}$ be two sequences of chains of the pseudo-arc $P$ such that,

(1) for each $i=0,1, \ldots, C_{i}$ and $D_{i}$ has the same number of links, so they are written as $C_{i}=\left\{C_{i}(0), \ldots C_{i}\left(n_{i}\right)\right\}$ and $D_{i}=\left\{D_{i}(0), \ldots D_{i}\left(n_{i}\right)\right\}$,

(2) $\lim _{i \rightarrow \infty} \operatorname{mesh} C_{i}=\lim _{i \rightarrow \infty} \operatorname{mesh} D_{i}=0$, and 
(3) for each $i$, there exists a pattern $f_{i}:\left\{0,1, \ldots, n_{i+1}\right\} \rightarrow\left\{0, \ldots n_{i}\right\}$ such that $C_{i+1}$ follows $f_{i}$ in $C_{i}$ and $D_{i+1}$ follows $f_{i}$ in $D_{i}$.

Then there exists a homeomorphism $h: P \rightarrow P$ such that, if $\operatorname{st}\left(p, C_{i}\right) \subset\left\{C_{i}(j), C_{i}(j+1)\right\}$, then $h(x) \in D_{i}(j) \cup D_{i}(j+1)$, for each $j=0, \ldots, n_{i}$ and for each $i=0,1, \ldots$

REMARK. In [5], a stronger condition

(2') $\operatorname{mesh} C_{i}<1 / i$ and $\operatorname{mesh} D_{i}<1 / i$

is required instead of the condition (2) above. The proof of the theorem easily shows that the same conclusion holds under the condition (2) above.

COROLLARY 1.6. Under the above notation, assume further that a point $p \in P$ satisfies $p \in C_{i}(0) \cap D_{i}(0)$ for each $i$. Then the above homeomorphism $h: P \rightarrow P$ satisfies $h(p)=p$.

Proof. Since $h(p) \in D_{i}(0) \cup D_{i}(1)$, we have $d(p, h(p)) \leq \operatorname{mesh} D_{i} \rightarrow 0$ as $i \rightarrow \infty$. The conclusion follows immediately.

2. Proof of Main theorem. Recall [8] that $C_{0}(L)$ is almost positive transitive if, for each pair of non-negative norm-one functions $f, g \in C_{0}(L)$ and for each $\epsilon>0$, there exists an isometry $T: C_{0}(L) \rightarrow C_{0}(L)$ such that $\|T f-g\|<\epsilon$. Also we say that $C_{0}(L)$ allows nearly polar decompositions if, for each $f \in C_{0}(L)$ and for each $\epsilon>0$, there exists an isometry $T: C_{0}(L) \rightarrow C_{0}(L)$ such that $\|f-T(|f|)\|<\epsilon$. It is easy to see that $C_{0}(L)$ is almost transitive if and only if it is almost positive transitive and allows nearly polar decompositions (cf. [8, p. 315]).

The following Lemma is implicitly stated in [8, Theorem 2], in which the proof is omitted. We give a proof here for completeness.

LEMMA 2.1. Let $L$ be a locally compact metrizable space such that the one-point compactification $\alpha L$ is metrizable with $\operatorname{dim} \alpha L=1$. Then $C_{0}(L)$ allows nearly polar decompositions.

Proof. Take an arbitrary $f \in C_{0}(L)$ and extend it canonically to the function on $\alpha L$ by defining $f(\infty)=0$. Since $\operatorname{dim} \alpha L=1$, one can show that, for each $\epsilon>0$, there exists a continuous function $f_{\epsilon}: \alpha L \rightarrow \mathbf{C}$ such that $\left\|f-f_{\epsilon}\right\|<\epsilon$ and $f_{\epsilon}(x) \neq 0$ for each $x \in \alpha L$ (see [4, Theorem 18], or [2, p. 76, 1.9.B]). Define $T: C_{0}(L) \rightarrow C_{0}(L)$ by

$$
(T g)(x)=\frac{f_{\epsilon}(x)}{\left|f_{\epsilon}(x)\right|} \cdot g(x), \quad g \in C_{0}(L) \text { and } x \in L .
$$

Clearly $T$ is an isometry. For an arbitrary $x \in L$, we estimate $|(T|f|)(x)-f(x)|$ as follows. Here the complex conjugate of $f(x)$ is denoted by $\overline{f(x)}$.

$$
\begin{aligned}
|(T|f|)(x)-f(x)|^{2} & =\left|\frac{f_{\epsilon}(x)}{\left|f_{\epsilon}(x)\right|} \cdot\right| f(x)|-f(x)|^{2} \\
& =2|f(x)|^{2}-|f(x)| \frac{f_{\epsilon}(x) \overline{f(x)}+\overline{f_{\epsilon}(x)} f(x)}{\left|f_{\epsilon}(x)\right|} \\
& =2|f(x)|\left(|f(x)|-\operatorname{Re} \frac{f_{\epsilon}(x) \overline{f(x)}}{\left|f_{\epsilon}(x)\right|}\right) \\
& \leq 2\|f\|\left(\frac{\left(\left|f_{\epsilon}(x)\right|+\epsilon\right)\left|f_{\epsilon}(x)\right|-\operatorname{Re} f_{\epsilon}(x) \overline{f(x)}}{\left|f_{\epsilon}(x)\right|}\right) \\
& =2\|f\|\left(\epsilon+\frac{\left|f_{\epsilon}(x)\right|^{2}-\operatorname{Re} f_{\epsilon}(x) \overline{f(x)}}{\left|f_{\epsilon}(x)\right|}\right) .
\end{aligned}
$$


Making use of the inequality $\left\|f-f_{\epsilon}\right\|<\epsilon$, it is easy to see

$$
\frac{\left|f_{\epsilon}(x)\right|^{2}-\operatorname{Re} f_{\epsilon}(x) \overline{f(x)}}{\left|f_{\epsilon}(x)\right|}<2 \epsilon .
$$

Thus we have $\|T|f|-f\|=\sup _{x \in L}|(T|f|)(x)-f(x)| \leq \sqrt{6\|f\| \epsilon}$, completing the proof.

Proof of Main Theorem. Let $P$ be the pseudo-arc and take a point $p \in P$. We show that $L=P \backslash\{p\}$ is an almost Wood space. Clearly $\alpha L \cong P$, a compact connected metrizable space and $\operatorname{dim} P=1$. Thus $C_{0}(L)$ allows nearly polar decompositions by Lemma 2.1. Hence it suffices to verify the conditions required in Theorem 1.1, yet it is more convenient to prove directly the almost positive transitivity of $C_{0}(L)$, along with exactly the same idea as that of [8, Theorem 3].

Take arbitrary pair of non-negative, norm-one functions $f, g \in C_{0}(L)$ and extend them to functions on $\alpha L=P$ by defining $f(p)=g(p)=0$. Then $f(\alpha L)=g(\alpha L)=[0,1]$. Fix a positive integer $n$ and let $I_{i}=\left(\frac{i}{n}-\frac{1}{4 n}, \frac{i+1}{n}+\frac{1}{4 n}\right) \cap[0,1]$ for $i=0, \ldots, n-1$. Let $C_{0}(i)=f^{-1}\left(I_{i}\right)$ and $D_{0}(i)=g^{-1}\left(I_{i}\right)$ for $i=0, \ldots, n-1 . C_{0}=\left\{C_{0}(0), \ldots, C_{0}(n-1)\right\}$ and $D_{0}=\left\{D_{0}(0), \ldots, D_{0}(n-1)\right\}$ are chains of $P$. As $f(p)=0$, we have $p \in C_{0}(0)$ and similarly $p \in D_{0}(0)$.

Take a small $0<\epsilon_{1}<1 / 2$ so that every subset $E$ of $P$ with $\operatorname{diam} E<\epsilon_{1}$ is contained in a link of $D_{0}$. By Theorem 1.3, there exists a chain $D_{1}=\left\{D_{1}(0), \ldots, D_{0}\left(n_{1}\right)\right\}$ with mesh $D_{1}<\epsilon_{1}$ such that $p \in D_{1}(0)$. Note that, for each link $D_{1}(i)$, there exists a link of $D_{0}$ containing $D_{1}(i)$. Hence there exists a pattern which $D_{1}$ follows in $D_{0}$. Let $f_{1}:\left\{0, \ldots, n_{1}\right\} \rightarrow\{0, \ldots, n\}$ be a such pattern. Apply Theorem 1.4 to take a chain $C_{1}=\left\{C_{1}(0), \ldots, C_{1}\left(n_{1}\right)\right\}$ which follows $f_{1}$ in $C_{0}$ such that $p \in C_{1}(0)$.

Take a small $0<\epsilon_{2}<1 / 3$ so that every subset $F$ of $P$ with $\operatorname{diam} F<\epsilon_{2}$ is contained in a link of $C_{1}$. Again by Theorem 1.3, there exists a chain $C_{2}=\left\{C_{2}(0), \ldots, C_{2}\left(n_{2}\right)\right\}$ with mesh $C_{2}<\epsilon_{2}$ such that $p \in C_{2}(0)$. Let $f_{2}:\left\{0, \ldots, n_{2}\right\} \rightarrow\left\{0, \ldots, n_{1}\right\}$ be a pattern which $C_{2}$ follows in $C_{1}$. We make another application of Theorem 1.4 to obtain a chain $D_{2}=\left\{D_{2}(0), \ldots, D_{2}\left(n_{2}\right)\right\}$ which follows $f_{2}$ in $D_{1}$ such that $p \in D_{2}(0)$. Note that $\operatorname{mesh} D_{2} \leq \operatorname{mesh} D_{1}<1 / 2$.

Continuing this process, we obtain sequences of chains $\left\{C_{i} \mid i=0,1, \ldots\right\}$ and $\left\{D_{i} \mid i=0,1, \ldots\right\}$ together with a sequence $\left\{f_{i}:\left\{0, \ldots, n_{i}\right\} \rightarrow\left\{0, \ldots, n_{i-1}\right\} \mid i=1,2, \ldots\right\}$ of patterns $\left(n_{0}=n\right)$ which satisfy the hypothesis of Theorem 1.5. Moreover the sequences have an additional property: $p \in \cap_{i=0}^{\infty} C_{i}(0) \cap \cap_{i=0}^{\infty} D_{i}(0)$. By Theorem 1.5 and Corollary 1.6, there exists a homeomorphism $\varphi: P \rightarrow P$ with $\varphi(p)=p$ such that, if $\operatorname{st}\left(x, C_{0}\right) \subset\left\{C_{0}(j), C_{0}(j+1)\right\}$, then $\varphi(x) \in D_{0}(j) \cup D_{0}(j+1)$.

Take a point $x \in C_{0}(j)$. Then by the definition of $C_{0}, f(x) \in\left(\frac{j}{n}-\frac{1}{4 n}, \frac{j+1}{n}+\frac{1}{4 n}\right)$. Since $\varphi(x) \in D_{0}(j) \cup D_{0}(j+1)$, we have similarly $g(\varphi(x)) \in\left(\frac{j}{n}-\frac{1}{4 n}, \frac{j+2}{n}+\frac{1}{4 n}\right)$. Hence $|f(x)-g(\varphi(x))| \leq \frac{2}{n}+\frac{1}{2 n}=\frac{5}{2 n}$. Since $x$ is an arbitrary point of $L$, we have $\|f-T g\| \leq$ $\frac{5}{2 n}$. For a given $\epsilon>0$, we may take $n$ so that $\frac{5}{2 n}<\epsilon$ to complete the proof of the almost positive transitivity of $C_{0}(L)$.

This completes the proof of Main Theorem.

\section{REFERENCES}

1. R. H. Bing, A homogeneous indecomposable plane continuum, Duke Math. J. 15(1948), $729-742$. 
2. R. Engelking, Theory of dimensions, finite and infinite, Sigma series in Pure Math $\mathbf{1 0}$ (Helderman Verlag, 1995).

3. P. Greim and M. Rajagopalan, Almost transitivity in $C_{0} L$, Math. Proc. Camb. Phil. Soc. 121 (1997), 75-80.

4. A. Jiménez-Vargas, J. F. Mena-Jurado and J. C. Navarro-Pascual, Mappings without fixed or antipodal points. Some geometric applications, Math. Scand. 84 (1999), 179-194.

5. W. Lewis, The Pseudo-arc, in Continuum theory and dynamical system (Arcata CA, 1989), Contemp. Math. 117 (1991), 103-123.

6. E. E. Moise, An indecomposable plane continuum which is homeomorphic to each of its nondegenerate subcontinua, Trans. Amer. Math. Soc. 63 (1948), 581-594.

7. F. C. Sánches, Transitivity of $M$-spaces and Wood's conjecture, Math. Proc. Camb. Phil. Soc. 124 (1998), 513-520.

8. F. C. Sánches, The covering dimension of Wood spaces, Glasgow Math. J. 44 (2002), 311-316.

9. G. V. Wood, Maximal symmetry in Banach spaces, Proc. Royal Irish Acad. 82A (1982), $177-186$. 